\title{
Weak Gravitational Lensing by Bocharova-Bronnikove-Melnikov-Bekenstein Black Holes Using Gauss-bonnet Theorem
}

\author{
Wajiha Javed, ${ }^{1, *}$ Muhammad Bilal Khadim, ${ }^{1, \dagger}$ and Ali Övgün ${ }^{2, \ddagger}$ \\ ${ }^{1}$ Division of Science and Technology, University of Education, Township-Lahore, Pakistan
}

\begin{abstract}
In this article, we demonstrate the weak gravitational lensing in the context of BocharovaBronnikove-Melnikov-Bekenstein (BBMB) black hole. To this desire, we derive the deflection angle of light in a plasma medium by BBMB black hole using the Gibbons and Werner method. First, we obtain the Gaussian optical curvature and implement the Gauss-Bonnet theorem to investigate the deflection angle for spherically symmetric spacetime of BBMB black hole. Moreover, we also analyze the graphical behavior of deflection angle by BBMB black hole in the presence of plasma medium.
\end{abstract}

PACS numbers: 95.30.Sf, 98.62.Sb, 97.60.Lf

Keywords: weak gravitational lensing; black hole; deflection angle; Gauss-Bonnet theorem

\section{INTRODUCTION}

The discovery of first neutron collision [1] was an epochal which is the beginning of gravitational wave astronomy. After gravitational waves was discovered, a large number of the modified gravity theories face a lot of problems. On the other hand, one of the important testing method of gravity is gravitational lensing. The light is deflected in the presence of a massive body which is predicted by General Relativity. This phenomena is called gravitational lensing. Its experimental conformation was first given by Cavendish, Soldner [2] as well as Einstein in their observation to identify the gravitational deflection of light. The gravitational lensing has been classified in the literature as a strong lensing, weak lensing and micro lensing [3,4].

Weak gravitational lensing is a powerful tool to measure masses of a variety of objects in the universe. The weak gravitational lensing provides a way to find the mass of astronomical objects without requiring about their composition or dynamical states. Weak lensing also investigate the cause of the accelerated expansion of the universe and also distinguish between modified gravity and dark energy. For instance, the lens equation is obtained for the Schwarzschild black hole by Ellis and Virbhadra [5] and more comprehensively discussed by Virbhadra [6]. Many authors investigate the gravitational lensing by other astrophysical objects such as naked singularities black holes $(\mathrm{BH})$, wormholes and few other related objects [7]-[29].

Gibbons and Werner (GW) proposed a new method to calculate weak deflection angle using the Gauss-Bonnet theorem (GBT) [30]. They showed that this method provides that the deflection angle is a global property. Sakalli and Ovgun applied this novel method to the Rindler modified [31] Schwarzschild black hole and obtein the weak deflection angle. The Gibbons-Werner method has been applied by different authors on black holes as well as wormholes [32]-[58]. The GBT method is based on the following equation [30]:

$$
\alpha=-\iint_{D \infty} \mathcal{K} d S
$$

Here, $\mathcal{K}$ and $d S$ represents the Gaussian curvature and surface element respectively. After that, Werner [59] extended this method and obtained the weak deflection of light by Kerr-Newman black hole by using the Nazims's method for Rander-Finsler metric. Recently, Gallo and Crisnejo (GC) [58] discussed the deflection of light in the plasma medium. Since then the work of weak gravitational lensing is continuously through the method of GW by using the GBT for different black holes and wormholes.

In this article, our main aim is to investigate weak deflection angle of BBMB black hole. For this purpose, this article is categories as follows; In section 2 we review the BBMB black hole. In section 3, by using the GBT we compute the weak deflection angle of BBMB black hole in plasma medium. In section 4 , we conclude our results.

\footnotetext{
*Electronic address: wajiha.javed@ue.edu.pk

†Electronic address: blaljutt723@gmail.com

‡Electronic address: ali.ovgun@emu.edu.tr
} 


\section{BOCHAROVA-BRONNIKOVE-MELNIKOV-BEKENSTEIN BLACK HOLE}

The BBMB black hole in a static and spherically symmetric form is given as [60]

$$
d s^{2}=-f(r) d t^{2}+\frac{d r^{2}}{f(r)}+r^{2}\left(d \theta^{2}+\sin ^{2} \theta d \Omega^{2}\right),
$$

where the metric function $f(r)$ is

$$
f(r)=1-\frac{2 m}{r}+\frac{m^{2}}{r^{2}}
$$

here $m$ is a mass of $\mathrm{BH}$. The optical spacetime in equatorial plane $\left(\theta=\frac{\pi}{2}\right)$ to get null geodesic $(d s=0)$

$$
d t^{2}=\frac{d r^{2}}{f(r)^{2}}+\frac{r^{2} d \Omega^{2}}{f(r)}
$$

To investigate the weak gravitational lensing by BBMB black hole in plasma medium, we calculate the refractive index $n(r)$ :

$$
n(r)=\sqrt{1-\frac{\omega_{e}^{2}}{\omega_{\infty}^{2}} f(r)}
$$

where $\omega_{e}$ and $\omega_{\infty}$ are electron plasma frequency and light frequency calculated by an observer at infinity respectively, then the corresponding optical metric illustrated as

$$
d t^{2}=g_{i j}^{o p t} d x^{i} d x^{j}=n^{2}(r)\left[-f(r) d t^{2}+\frac{d r^{2}}{f(r)}+r^{2}\left(d \Omega^{2}\right)\right] .
$$

The Gaussian optical curvature that is evaluated as follows:

$$
\mathcal{K}=\frac{\text { RicciScalar }}{2}
$$

After simplifying, Gaussian optical curvature for BBMB black hole in leading order term is calculated as follow:

$$
\mathcal{K} \approx-\frac{2 m}{r^{3}}-\frac{3 m \omega_{e}^{2}}{\omega_{\infty}^{2} r^{3}}+\frac{6 m^{2}}{r^{4}}+\frac{17 m^{2} \omega_{e}^{2}}{r^{4} \omega_{\infty}^{2}}
$$

\section{DEFLECTION ANGLE OF BBMB BLACK HOLES AND GAUSS-BONNET THEOREM}

In this section, we drive the deflection angle of light by BBMB BH using the GBT. By using GBT in the region $\mathcal{H}_{R}$, given as [30]

$$
\int_{\mathcal{H}_{R}} \mathcal{K} d S+\oint_{\partial \mathcal{H}_{R}} k d t+\sum_{t} \hat{\alpha}=2 \pi \mathcal{X}\left(\mathcal{H}_{R}\right)
$$

where, $k$ represent the geodesic curvature, and $\mathcal{K}$ denotes the Gaussian optical curvature and one can define $k$ as $k=\bar{g}\left(\nabla_{\hat{\alpha}} \hat{\alpha}, \check{\alpha}\right)$ in such a way that $\bar{g}(\hat{\alpha}, \hat{\alpha})=1$, where $\hat{\alpha}$ represent the unit acceleration vector and $\alpha_{t}$ denotes the exterior angle at $t^{t h}$ vertex respectively. As $R \rightarrow \infty$, we obtain the jump angles equal to $\pi / 2$, hence $\alpha_{O}+\alpha_{S} \rightarrow \pi$. Here, $\mathcal{X}\left(\mathcal{H}_{R}\right)=1$ is a Euler characteristic number and $\mathcal{H}_{R}$ denotes the non-singular domain. Therefore, we obtain [30]

$$
\iint_{\mathcal{H}_{R}} \mathcal{K} d S+\oint_{\partial \mathcal{H}_{R}} k d t+\hat{\alpha}=2 \pi \mathcal{X}\left(\mathcal{H}_{R}\right)
$$


where, the total jump angle is $\hat{\alpha}=\pi$. At $0^{t h}$ order weak field deflection limit of the light is defined as $r(t)=b / \sin \phi$, and GW shows that the above equation for GBT reduces to simple form [30], then GC analyses the deflection angle in the plasma medium by using this equation [58]:

$$
\alpha=-\int_{0}^{\pi} \int_{b / \sin \phi}^{\infty} \mathcal{K} \sqrt{\operatorname{det} \bar{g}} d u d \phi
$$

here we put the leading term of equation Eq. 7 into above equation Eq. 10, so the obtained deflection angle of the photon rays are moving in a medium of homogeneous plasma up to leading order term is computed as:

$$
\sigma \simeq \frac{4 m}{b}-\frac{3 m^{2} \pi}{2 b^{2}}+\frac{6 m^{2} \omega_{e}^{2}}{b \omega_{\infty}^{2}}-\frac{17 m^{2} \pi \omega_{e}^{2}}{4 b^{2} \omega_{\infty}^{2}}
$$

Hence, the effect of plasma can be removed if $\left(\omega_{e}=0\right)$, or $\left(\omega_{e} / \omega_{\infty} \rightarrow 0\right)$, and it reduces to vacuum case. The value of the photon frequency in plasma medium is $\omega_{e} / \omega_{\infty}=6 \times 10^{-3}$ [61].

\section{CONCLUSION}

The present article is about the investigation of deflection angle by BBMB black hole in plasma medium. In this regard, we analysis the weak gravitational lensing by using GBT and get the deflection angle of light for BBMB black hole.
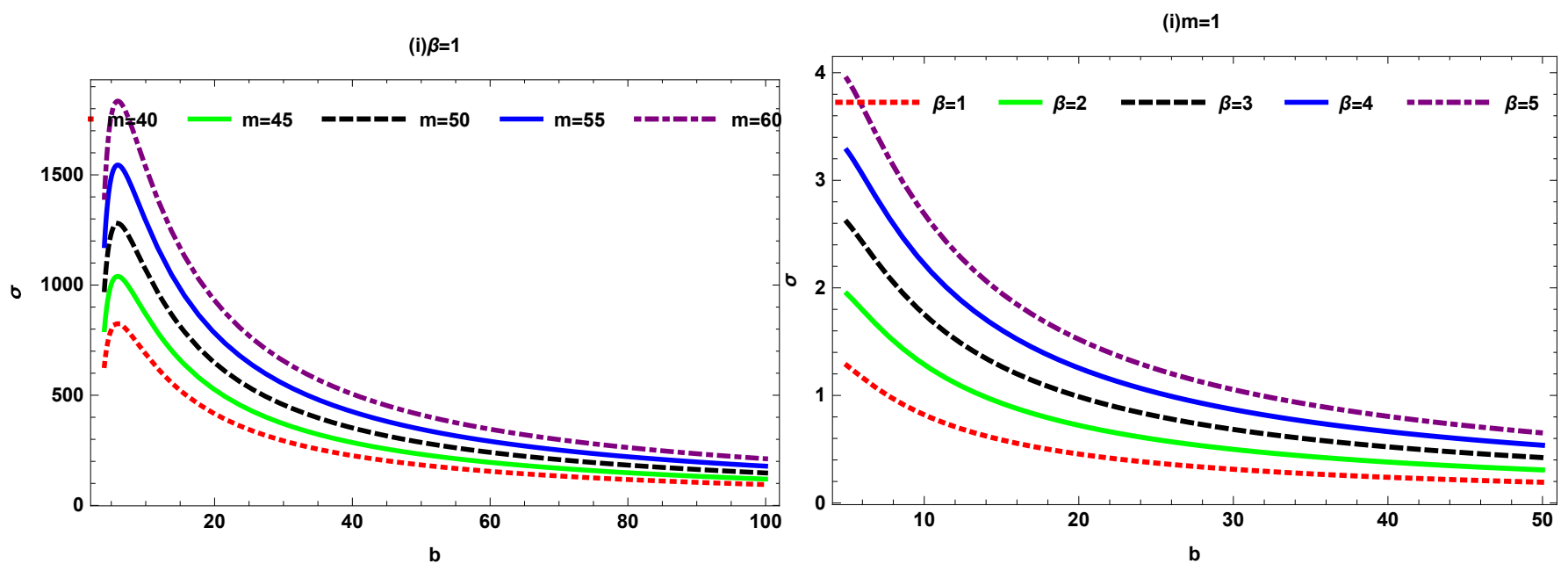

Figure 1: $\sigma$ versus $b$.

- Figure 1 indicates the behavior of deflection angle w.r.t $b$ by fixing the value of $\beta$ and varying $m$. It is to be observed that for values of $m$ and $\beta>0$ the behavior of deflection angle gradually increasing and then suddenly the deflection angle gradually decreasing. For values of $m$ and taking $\beta \leq 0$ we noticed that the deflection angle gradually positively decreasing.

- Figure 2 represents the behavior of deflection angle w.r.t $b$ by varying the mass $\beta$ and taking $m$ fixed. We noticed that for values of $\beta>0$ the behavior of deflection angle gradually increasing, on the other hand for the values of $\beta<0$ the behavior of deflection angle negatively decreasing. But for value of $b<5$ the behavior of deflection angle overlap.

The obtained deflection angle is given as follows:

$$
\sigma \simeq \frac{4 m}{b}-\frac{3 m^{2} \pi}{2 b^{2}}+\frac{6 m^{2} \omega_{e}^{2}}{b \omega_{\infty}^{2}}-\frac{17 m^{2} \pi \omega_{e}^{2}}{4 b^{2} \omega_{\infty}^{2}}
$$

We examine that by the reduction of some parameters the obtained deflection angle converted into the 
Schwarzschild deflection angle up to the first order terms. In addition we also discuss the graphical effect of different parameters on deflection angle by BBMB in a plasma medium.

[1] B. P. Abbott et al. [LIGO Scientific and Virgo Collaborations], Phys. Rev. Lett. 116, no. 6, 061102 (2016).

[2] J.Soldner, in Astronomisches Jahrbuch fur 1804, p. 161 (1801)

[3] M. Bartelmann and P. Schneider, Phys. Rept. 340, 291 (2001).

[4] P. V. P. Cunha, C. A. R. Herdeiro, E. Radu and H. F. Runarsson, Phys. Rev. Lett. 115, no. 21, 211102 (2015).

[5] K.S. Virbhadra and G. F. R. Ellis, Phys. Rev. D.bf62, 084003 (2000).

[6] K.S. Virbhadra, Phys. Rev. D 79, 083004 (2009).

[7] M. Bartelmann, Class. Quant. Grav. 27, 233001 (2010).

[8] C. R. Keeton, C. S. Kochanek and E. E. Falco, Astrophys. J. 509, 561 (1998).

[9] V. Bozza, Phys. Rev. D 66, 103001 (2002).

[10] S. b. Chen and J. 1. Jing, Phys. Rev. D 80, 024036 (2009).

[11] E. F. Eiroa, G. E. Romero and D. F. Torres, Phys. Rev. D 66, 024010 (2002).

[12] A. Bhadra, Phys. Rev. D 67, 103009 (2003).

[13] R. Whisker, Phys. Rev. D 71, 064004 (2005).

[14] K. K. Nandi, Y. Z. Zhang and A. V. Zakharov, Phys. Rev. D 74, 024020 (2006).

[15] S. Mao and B. Paczynski, Astrophys. J. 374, L37 (1991).

[16] H. Hoekstra, H. K. C. Yee and M. D. Gladders, Astrophys. J. 606, 67 (2004).

[17] K. S. Virbhadra and G. F. R. Ellis, Phys. Rev. D 65, 103004 (2002).

[18] O. Gurtug and M. Mangut, Phys. Rev. D 99, no. 8, 084003 (2019)

[19] K. S. Virbhadra and G. F. R. Ellis, Phys. Rev. D 62, 084003 (2000).

[20] O. Kasikci and C. Deliduman, Phys. Rev. D 100, no. 2, 024019 (2019)

[21] E. Gallo and O. M. Moreschi, Phys. Rev. D 83, 083007 (2011).

[22] G. Crisnejo and E. Gallo, Phys. Rev. D 97, no. 8, 084010 (2018).

[23] M. Sharif and S. Iftikhar, Astrophys. Space Sci. 357, no. 1, 85 (2015).

[24] G. W. Gibbons, Phys. Lett. B 308, 237 (1993).

[25] A. Edery and M. B. Paranjape, Phys. Rev. D 58, 024011 (1998).

[26] J. Bodenner and C. Will, Am. J. Phys. 71, 770 (2003).

[27] K. Nakajima and H. Asada, Phys. Rev. D 85, 107501 (2012).

[28] W. G. Cao and Y. Xie, Eur. Phys. J. C 78, 191 (2018).

[29] C. Y.Wang, Y. F. Shen and Y. Xie, J. Cosmol. Astropart. Phys. 04 (2019) 022.

[30] G. W. Gibbons and M. C. Werner, Classical Quantum Gravity 25, 235009 (2008).

[31] I. Sakalli, A. Ovgun, EPL 118(6), 60006 (2017).

[32] K. Jusufi, I. Sakalli, and A. Övgün, Phys. Rev. D 96, 024040 (2017).

[33] Z. Li and A. Övgün, Phys. Rev. D 101, no. 2, 024040 (2020)

[34] K. Jusufi and A. Övgün, Phys. Rev. D 97, 024042 (2018).

[35] Y. Kumaran and A. Övgün, Chin. Phys. C 44, 025101 (2020).

[36] K. Jusufi, A. Övgün, J. Saavedra, Y. Vasquez, and P. A. Gonzalez, Phys. Rev. D 97, 124024 (2018).

[37] Z. Li, G. He and T. Zhou, Phys. Rev. D 101, no. 4, 044001 (2020).

[38] K. Jusufi and A. Övgün, Int. J. Geom. Meth. Mod. Phys. 16, no. 08, 1950116 (2019)

[39] A. Övgün, G. Gyulchev, and K. Jusufi, Annals Phys. 406, 152 (2019).

[40] K. Jusufi and A. Övgün, Phys. Rev. D 97, 064030 (2018).

[41] Z. Li and T. Zhou, Phys. Rev. D 101, no. 4, 044043 (2020)

[42] K. Jusufi, A. Övgün, A. Banerjee and I. Sakalli, Eur. Phys. J. Plus 134, no. 9, 428 (2019).

[43] Z. Li and J. Jia, Eur. Phys. J. C 80, no. 2, 157 (2020)

[44] K. Jusufi, M. C. Werner, A. Banerjee, and A. Övgün, Phys. Rev. D 95, 104012 (2017).

[45] A. Övgün, K. Jusufi, and I. Sakalli, Ann. Phys. (Amsterdam) 399, 193 (2018).

[46] A. Övgün, K. Jusufi, and I. Sakalli, Phys. Rev. D 99, 024042 (2019).

[47] A. Övgün, I. Sakalli, and J. Saavedra, JCAP 1810, 041 (2018).

[48] A. Övgün, Universe 5, 115 (2019).

[49] A. Övgün, Phys. Rev. D 98, 044033 (2018).

[50] A. Övgün, I. Sakalli, and J. Saavedra, Annals Phys. 411, 167978 (2019).

[51] A. Övgün, Phys. Rev. D 99, 104075 (2019).

[52] W. Javed, R. Babar, and A. Övgün, Phys. Rev. D 99, 084012 (2019).

[53] W. Javed, R. Babar, and A. Övgün, Phys. Rev. D 100, 104032 (2019).

[54] W. Javed, j. Abbas and A. Övgün, Phys. Rev. D 100, no. 4, 044052 (2019).

[55] W. Javed, J. Abbas, A. Övgün, Eur. Phys. J. C 79, 694 (2019).

[56] W. Javed, M. Bilal Khadim, J. Abbas, A. Övgün, Eur. Phys. J. Plus 135, 314 (2020).

[57] A. Ishihara, Y. Suzuki, T.Ono,T. Kitamura, H.Asada, Phys. Rev. D 94(8), 084015 (2016) 
[58] G. Crisnejo, E. Gallo, Phys. Rev. D 97(12), 124016 (2018)

[59] M. C. Werner, Gen. Rel. Grav. 44, 3047 (2012).

[60] E.W Maxwell, arXiv:2001.01351v1 [gr-qc].

[61] G. S. Bisnovatyi-Kogan and O. Y. Tsupko, Mon. Not. Roy. Astron. Soc. 404, 1790 (2010). 